\title{
Evaluation on Development Capacity of Overseas Chinese Enterprises Based on Improved BP Neural-Networks
}

\author{
Ke MA ${ }^{1, *}$ and Xian-shui SUN ${ }^{2}$ \\ ${ }^{1}$ Department of PE and Military Training, Zhejiang Sci-Tech University, Hangzhou 310018, China \\ ${ }^{2}$ School of Informatics and Electronics, Zhejiang Sci-Tech University, Hangzhou 310018, China \\ ${ }^{*}$ Corresponding author
}

Keywords: Overseas Chinese enterprises, Development capacity, BP neural-networks, Learning algorithm.

\begin{abstract}
An approach based on improved BP neural-networks is proposed for the evaluation on the development capacity of overseas Chinese enterprise. In order to cope with the slow learning speed and local minimal problems of BP neural-networks, an adaptive learning rate selection method based errors is presented. According to the features of overseas Chinese enterprise, the parameters of input level and number of neurons of hidden level of the BP neural-networks are investigated. Finally, the objective and quantified evaluation result can be achieved from the output neuron.
\end{abstract}

\section{基于改进型 $\mathrm{BP}$ 神经网络的侨资企业发展能力评价 马可 ${ }^{1, *}$, 孙显水 ${ }^{2}$ \\ 1浙江理工大学体军部, 杭州 310018, 中国 \\ 2浙江理工大学信息学院, 杭州 310018, 中国 通讯作者}

关键词：侨资企业; 发展能力; BP神经网络; 学习算法

中文摘要. 构建了一种改进型的BP神经网络对侨资企业的发展能力进行评价。针对BP神经网 络学习速度慢、易陷入局部极小的特点, 提出了学习步长随学习误差而自适应调节的学习算 法。结合侨资企业的特点, 研究了神经网络输入节点所需要的指标体系, 隐含层神经元的数 目。最后在神经网络的输出节点得到侨资企业发展能力客观、量化的评价结果。

\section{1. 引言}

对企业进行发展能力评价是企业管理的核心内容之一。企业的发展能力是企业通过自身 的生产经营活动，不断扩大积累而形成的发展潜能[1]。

受全球金融危机的影响，投资中国的侨资企业经历了前所未有的震撼。由于经营领域、 经营状况不同，不同的侨资企业面临不同的压力[2]。总体而言，目前我国侨资企业主要面临 以下几方面的共性问题：一是在投资环境方面，“软件不软，硬件不硬”的问题在不少地区仍 在一定程度上存在[3]。重引进, 轻管理的现象在有些地区仍然存在, 一些涉及到侨商投诉得 不到有效解决的现象时有发生。另外, 有些窗口部门的办事效率不高也在一定程度上影响了 侨资企业的发展。二是企业融资困难, 这在一定程度上影响了企业转型升级。三是企业人才 基础薄弱，缺乏高质量的研发团队，企业创新能力受限。一个企业能否健康发展取决于多种 因素，包括外部经营环境，企业内在素质及资源条件等[1]。因此，结合侨资企业发展的各种 主要因素对其发展能力进行客观、量化的评价对于侨资企业的健康发展具有十分重要的意义。 
神经网络是人工智能领域的热点研究内容，是基于模仿人脑功能而形成的一种信息处理 系统。它由神经元及其互连组成网络结构。神经网络具有很好的鲁棒性和学习联想能力, 能 处理复杂的非线性问题。本文采用BP神经网络对侨资企业的发展能力进行评价。

\section{2. 用于本研究的BP神经网络模型}

用于侨资企业发展能力评价的BP神经网络的拓扑结构如图1所示。网络共有3层，采用前 馈连接方式。其中, 输入层神经元的数目对应于评价所采用的指标数(这些指标将在第 4 节中 介绍)。隐含层神经元数目的选取可参照经验公式 $\rho \leq \sqrt{M(N+3)}+1$ [4], 其中 $\rho$ 为最佳隐含层神 经元数目, $M$ 为样本数, $N$ 为神经网络输出层神经元数目。网络输出层只有一个神经元, 其 输出值代表了侨资企业发展能力评价的量化值。

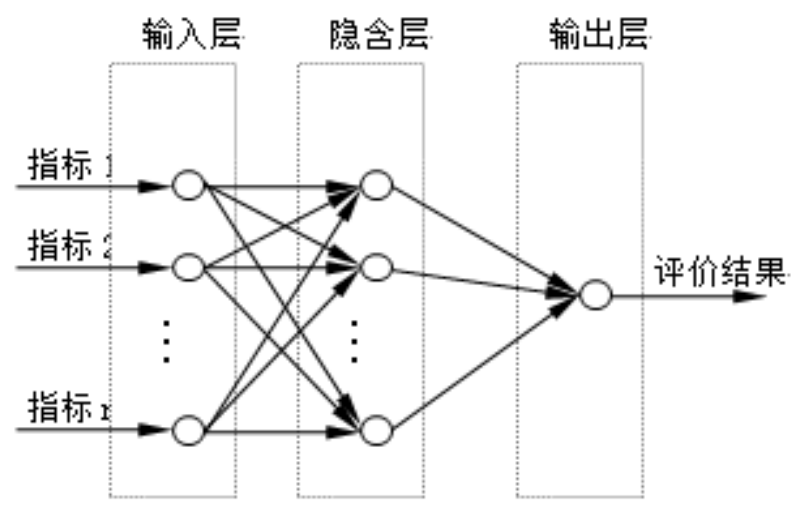

图1 用于本研究的BP网络结构

BP神经网络的训练样本由已知的指标集和所对应的发展能力评价结果构成。网络训练完 毕后, 只要在网络的输入层输入某侨资企业的 $n$ 个指标, 便可以在网络的输出层得到该企业发 展能力的量化评价结果。传统的BP神经网络采用误差反传（Back Propagation）学习方法, 其 连接权重 ${ }^{w_{i j}}$ 按下式更新:

$$
w_{i j}(t+1)=w_{i j}(t)+\eta \delta_{p j} O_{p j}
$$

其中, $w_{i j}(t+1)$ 为 $t+1$ 时刻的连接权重, $w_{i j}(t)$ 为 $t$ 时刻的连接权重, $\eta$ 为学习步长, $O_{p j}$ 定 义如下:

$$
O_{p j}=f\left(\sum_{i}\left(W_{j i} O_{p i}-\theta_{j}\right)\right)
$$

其中, $\theta_{j}$ 为神经元的阈值, $f(x)$ 为非线性函数, 一般取为 $\mathrm{S}$ 形函数, 即

$$
f(x)=\frac{1}{1+e^{-x}}
$$

(1)式中, $\delta_{p j}$ 为各层神经元的误差信号。

值得提出的是，传统BP神经网络的学习速度太慢，有时还会陷入局部极小的局面 $[5,6]$, 这在很大程度上限制了 $\mathrm{BP}$ 神经网络在侨资企业发展能力评价中的应用。所以为了使 $\mathrm{BP}$ 神经网 络适用于本文的研究, 必须对其学习算法做出改进。

\section{BP学习算法的改进}

在 $\mathrm{BP}$ 学习算法中, 我们希望学习步长 $\eta$ 能随误差 $E$ 的变化而变化, 即: 如果本次迭代的误 差呈减小趋势, 则增大 $\eta$, 以加快学习速度; 如果误差没有呈减小的趋势, 则减小 $\eta$, 抑制学 习速度。由此, 我们得出步长 $\eta$ 的调节规则: 
取 $\eta_{0}=0.3$

$\eta_{t}=\eta_{t-1}-\varphi \operatorname{tg} \frac{\pi\left(E_{t}-E_{t-1}\right)}{4\left(E_{t}+E_{t-1}\right)}, \varphi$ 为常数。

为使 $0<\eta<1$, 我们令

$\eta_{t}=\eta_{t-1}-\varphi \operatorname{tg} \frac{\pi\left(E_{t}-E_{t-1}\right)}{4\left(E_{t}+E_{t-1}\right)}<0$ 时, $\eta=0.3$,

$\eta_{t}=\eta_{t-1}-\varphi \operatorname{tg} \frac{\pi\left(E_{t}-E_{t-1}\right)}{4\left(E_{t}+E_{t-1}\right)}>1$ 时, $\eta=0.5$ 。

这种对 $\eta$ 不断调节的机制可以使神经网络收玫速度明显加快, 并有效克服局部极小问题 的产生。

\section{BP神经网络的输入层指标体系}

\section{1 指标体系的选择}

$\mathrm{BP}$ 神经网络输入层指标的选择是侨资企业发展能力有效评价的关键。本文对于这一指标 体系的选择遵从以下几个原则:

(1) 完备性原则。所选的指标要既能反映侨资企业内部发展状况, 又要考虑企业的外部 环境因素。

(2) 客观性原则。尽量选择客观性指标, 使根据BP神经网络计算所得到的评价结果更具 客观性和真实性。

(3) 去相关性原则。在指标选择时尽量选择统计上互不相关的指标, 这样既可以降低输 入层神经元的数目, 又可以提高评价的科学性。

根据以上的指标体系选择原则, 我们从侨资企业发展所涉及的外部影响力、创新投入、 发展效益、发展潜力、发展效率以及人员素质等要素出发, 确定了 18 个相对应的评价指标。 我们将这 18 个评价指标作为BP神经网络输入层神经元所对应的指标体系, 并对其进行了编 码, 如表1所示。

\section{2 指标体系的数据处理}

为了便于 $\mathrm{BP}$ 神经网络的计算分析, 以上所有指标都要进行归一化处理。此外, 尽管我们 在指标选择时尽量遵循了客观性原则, 但仍有少量指标难以数据量化, 如上表中的人员素质 指标等。对于这些指标可以通过群众测评、年度考核以及与个人相关的工作实绩相挂钩分析 得到。

表1 BP神经网络输入层指标体系及其代码

\begin{tabular}{|c|c|c|c|}
\hline & 评价要素 & 评价指标 & 指标代码 \\
\hline \multirow{10}{*}{$\begin{array}{l}\text { 经 } \\
\text { 网 } \\
\text { 络 } \\
\text { 输 } \\
\text { 入 } \\
\text { 层 } \\
\text { 指 } \\
\text { 标 } \\
\text { 体 } \\
\text { 系 }\end{array}$} & \multirow{3}{*}{$\begin{array}{c}\text { 外部影响 } \\
\text { 力 }\end{array}$} & 政府扶持力度 & $z_{1}$ \\
\hline & & 企业知名度 & $z_{2}$ \\
\hline & & 企业融资成本 & $z_{3}$ \\
\hline & \multirow{3}{*}{ 创新投入 } & R\&D投入占销售收入比率 & $z_{4}$ \\
\hline & & 研发人员素质 (学历比例) & $z_{5}$ \\
\hline & & 专利及专有技术拥有数 & $z_{6}$ \\
\hline & \multirow{3}{*}{ 发展效益 } & 经营净利率 & $z_{7}$ \\
\hline & & 资产利润率 & $z_{8}$ \\
\hline & & 净资产收益率 & $z_{9}$ \\
\hline & 发展潜力 & 净利润增长率 & $z_{10}$ \\
\hline
\end{tabular}




\begin{tabular}{|c|c|c}
\hline \multirow{2}{*}{} & 主营收入增长率 & $z_{11}$ \\
\cline { 2 - 3 } & 总资产增长率 & $z_{12}$ \\
\hline \multirow{4}{*}{ 人发展效率 } & 存贷周转率 & $z_{13}$ \\
\cline { 2 - 3 } & 应收账款周转率 & $z_{14}$ \\
\cline { 2 - 3 } & 专业知识技能 & $z_{15}$ \\
\cline { 2 - 3 } & 非专业技能 & $z_{16}$ \\
\cline { 2 - 3 } & 关系能力 & $z_{17}$ \\
\cline { 2 - 3 } & 心理素质 & $z_{18}$ \\
\hline
\end{tabular}

\section{4. 结束语}

随着国际全球化竞争的日趋激烈，进一步提升侨资企业的发展能力是当务之急。本文利 用BP神经网络技术，提出了一种侨资企业发展能力的客观评价方法。该方法对传统BP神经网 络进行了改进, 提出了新的网络学习算法, 提升了学习速度, 并可有效避免陷入局部极小。 由于BP神经网络具有很好的鲁棒性和学习联想能力, 能处理复杂的非线性问题, 所以利用这 一手段对侨资企业的发展能力进行评价能得到客观、量化的结果。本文的工作可为侨资企业 管理人员分析企业的发展能力提供借鉴，有助于侨资企业高层进行决策。

\section{致谢}

本文为浙江理工大学“521计划”资助项目的阶段性成果之一。

\section{References}

[1] X. Zhang, Prediction of Development Capacity of Enterprise using Neural-Networks, Communication of Finance and Accounting, vol.3, pp. 112-113, 2009.

[2] J. Zhao, Impression on some overseas Chinese enterprises in the world financial crisis, Overseas Chinese Journal of Bagui, vol.13, pp. 45-48, 2009.

[3] S. Liao, On overseas Chinese enterprises' challenge and countermeasures, Innovation, vol.3, pp. 56-57, 2009.

[4] X. Zhu and Y. Wang, Research on the evaluation of independent innovation capability among small and medium-sized enterprises in prospect of value based management, Science \& Technology Progress and Policy, vol.26, pp. 175-178, 2009.

[5] W. S. Stornetta and B. A. Huberman, An improved three-layer back propagation algorithm, Proceedings of the First IEEE International Conference on Neural Network3, vol. 2, pp. 637-643, 1987.

[6] W. Liao, Y. Chi and G. Wang, Predictive ability of BP neural network based on penalty coefficient, Journal of Liaoning Technical University, vol. 32, pp. 174-177, 2013. 